\title{
When does coarsening occur in the dynamics of one-dimensional fronts ?
}

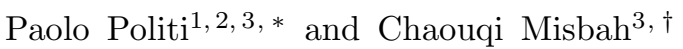 \\ ${ }^{1}$ Istituto di Fisica Applicata "N. Carrara", Consiglio Nazionale delle Ricerche, \\ Via Madonna del Piano, 50019 Sesto Fiorentino, Italy \\ ${ }^{2}$ Istituto Nazionale per la Fisica della Materia, UdR Firenze, \\ Via G. Sansone 1, 50019 Sesto Fiorentino, Italy \\ ${ }^{3}$ Laboratoire de Spectrométrie Physique-GREPHE, CNRS, \\ UJF-Grenoble 1, BP87, F-38402 Saint Martin d'Hères, France
}

(Dated: November 1, 2018)

\begin{abstract}
Dynamics of a one-dimensional growing front with an unstable straight profile are analyzed. We argue that a coarsening process occurs if and only if the period $\lambda$ of the steady state solution is an increasing function of its amplitude $A$. This statement is rigorously proved for two important classes of conserved and nonconserved models by investigating the phase diffusion equation of the steady pattern. We further provide clear numerical evidences for the growth equation of a stepped crystal surface.

PACS numbers: 05.70.Ln, 81.10.Aj, 05.45.-a, 68.35.Ct
\end{abstract}

Introduction - One of the most fascinating feature of nonequilibrium systems is their ability to build up a spatial (generally) ordered pattern from an initially structurless state when some control parameter reaches a critical value. Typical examples can be found in hydrodynamics, crystal growth, the Turing reaction-diffusion systems, and so on [1]. These pattern forming systems can be broadly classified into two important categories: (i) the first category includes the systems which select a typical pattern length scale $\lambda$ which is fixed for a given value of the control parameters. In that case the whole pattern needs not to be steady in time but can undergo oscillations, or even exhibit chaotic dynamics (a prominent example is the Kuramoto-Sivashinsky dynamics 2]). What matters is the selection of a length scale. (ii) The second category corresponds to the situation where there is a perpetual coarsening, in that the wavelength $\lambda(t)$ increases without bound in the course of time. Interrupted coarsening may also happen for special situations (see below). One must exclude the trivial case where the bifurcation occurs at a wavenumber $q=q_{c}$ and the band $\Delta q$ of active modes extends from $q_{c}-\delta_{1}$ to $q_{c}+\delta_{2}$. If $\Delta q=\delta_{1}+\delta_{2}$ is not too large, the system selects, of course, a pattern with a given intrinsic lengthscale. Examples include Turing patterns, Rayleigh-Bénard convection..., close enough to the instability threshold. Our discussion focuses on situations where there is no lower boundary on the active wavenumbers (arbitrarily long wavelengths are active). It is only at very large distance from the instability threshold that, for example, the Turing and Rayleigh-Bénard systems exhibit nontrivial dynamics (chaos, coarsening of turbulent plumes...), and this is precisely due to the fact that higher and higher harmonics become active.

A central question in nonequilibrium pattern forming systems is to identify general criteria (if any) for the presence of coarsening or not without resorting to a system- atic time-dependent calculation. We put forward a criterion for some classes of nonlinear equations which is uniquely based on the behavior of the steady-state solutions. This criterion is derived on the basis of the analysis of the phase diffusion equation [1], $\partial_{T} \psi=D \partial_{X X} \psi$, $\psi$ being the phase. A negative sign of the phase diffusion coefficient, $D$, is therefore a signature for the systems that undergo coarsening [3]. The second and more important step will be to relate the sign of $D$ with the derivative, $\partial \lambda / \partial A$, of the period $\lambda$ of the stationary solution with respect to its amplitude $A$. We will show that the sign of $D$ is opposite to that of $\partial \lambda / \partial A$. A coarsening process can therefore take place, $D<0$, if and only if $\partial \lambda / \partial A$ is positive. This relation will be proved analytically for two classes of nonconserved and conserved equations (Eqs. (1) and (10) respectively) and checked numerically for an equation which can display different dynamical scenarios with varying some physical parameters (Eq. (14)). Finally, considerations based on the Lyapunov functional will complement our analysis.

Nonconserved equations - We begin with the following class of nonconserved equations [4]:

$$
\partial_{t} u=\partial_{x}^{2} u-F(u) \equiv L[u],
$$

where $\partial_{t}, \partial_{x}$ indicate the time and the spatial derivatives, respectively. $F(u)$ is a generic function, playing the role of a force. We require that $F(u) \approx-u$ at small $u$, so the trivial solution $u(x, t)=0$ is linearly unstable against fluctuations of wavelength $\lambda>\lambda_{0}=2 \pi$. The GinzburgLandau (GL) equation is recovered for $F(u)=-u+u^{3}$, so Eq. (11) will be referred to as the generalized GL equation.

We can now remark that stationary solutions, $u_{0}(x)$, satisfy the condition

$$
\partial_{x}^{2} u_{0}-F\left(u_{0}\right)=0
$$

and $u_{0}(x)$ corresponds to the motion of a particle in the potential $V(u)=-\int d u F(u)$. For a linearly unstable profile, $V(u) \approx u^{2} / 2$ at small $u$. 
The steady-state $u_{0}(x)$ is a periodic solution with the spatial periodicity $\lambda, u_{0}(x+\lambda)=u_{0}(x)$. It is useful to consider the phase of the pattern, $\phi$, and we shall use a nonlinear WKB-like analysis [1] for the derivation of the phase equation. For a steady-state periodic solution, $\phi=q x$ where $q=2 \pi / \lambda$. In general the pattern will evolve in time, and thus the phase $\phi(x, t)$ is a function of space and time. We consider long wavelength modulations (which are the most dangerous ones owing to translational invariance of the initial pattern), and let $\epsilon$ denote the smallness of the phase modulation. We introduce a slow phase $\psi$ which is a function of a slow spatial variable $X \equiv \epsilon x$. Because we expect any deviation from the periodic pattern to evolve diffusively we anticipate that the slow time $T$ scales as $T=\epsilon^{2} t$. The slow phase $\psi(X, T)$ is related to the fast phase $\phi(x, t)$ by $\phi=\psi / \epsilon$ so that the local wavenumber is given by $q(X, T)=\partial \phi / \partial x=\partial \psi / \partial X$. In a multiscale spirit we have to make the substitutions

$$
\partial_{x} \rightarrow q \partial_{\phi}+\epsilon \partial_{X}, \quad \partial_{t} \rightarrow \epsilon^{2} \partial_{T} \phi \partial_{\phi}+\epsilon^{2} \partial_{T} .
$$

We then expand $u=u_{0}+\epsilon u_{1}+\ldots$ in powers of $\epsilon$. To leading order $\epsilon^{0}$ we find that $u_{0}$ obeys $L\left[u_{0}\right]=0$. For the generalized GL equation we have

$$
q^{2} \partial_{\phi \phi} u_{0}-F\left(u_{0}\right)=0 .
$$

The next order reads $\tilde{L}\left[u_{1}\right]=f\left(u_{0}\right)$, where $\tilde{L}$ is the (Fréchet) derivative [5] of $L$ and $f$ is the inhomogeneous term arising from phase modulation. For the generalized GL equation, $\tilde{L}=q^{2} \partial_{\phi \phi}-F^{\prime}\left(u_{0}\right)$, and

$$
f\left(u_{0}\right)=\partial_{T} \psi \partial_{\phi} u_{0}-\left[\partial_{\phi} u_{0}+2 q \partial_{\phi q} u_{0}\right] \partial_{X X} \psi .
$$

It is easily shown that, because of translational invariance, the linear operator $\tilde{L}$ has the nontrivial solution $\partial_{\phi} u_{0}$. It follows that $u_{1}$ exists only if the inhomogeneous term $f\left(u_{0}\right)$ is orthogonal to $\partial_{\phi} u_{0}[\underline{6}]$. This condition results in the sought after diffusion equation

$$
\partial_{T} \psi=D \partial_{X X} \psi,
$$

where $D$, the phase diffusion coefficient, is given by

$$
D=\frac{\partial_{q}\left\langle q\left(\partial_{\phi} u_{0}\right)^{2}\right\rangle}{\left\langle\left(\partial_{\phi} u_{0}\right)^{2}\right\rangle} \equiv \frac{D_{1}}{D_{2}} .
$$

Here above, $\langle\ldots\rangle=(2 \pi)^{-1} \int_{0}^{2 \pi} \ldots d \phi$ is the inner product, the denominator $D_{2}$ is always positive and the sign of $D$ is fixed by the numerator $D_{1}=\partial_{q}\left\langle q\left(\partial_{\phi} u_{0}\right)^{2}\right\rangle$.

Eq. (4), which is equivalent to Eq. (2), corresponds to the equation of motion of a particle of unitary mass, subject to the force $F\left(u_{0}\right): u_{0}$ is the spatial coordinate and $\tau=\phi / q$ is the time. So, we have:

$$
\left\langle q\left(\partial_{\phi} u_{0}\right)^{2}\right\rangle=\frac{1}{2 \pi} \int_{0}^{2 \pi / q} d \tau\left(\partial_{\tau} u_{0}\right)^{2}=\frac{J}{2 \pi},
$$

where we have introduced the action variable $J$.

Finally, if we remember [7] that the period of the oscillatory motion is given by the derivative of the action $J$ with respect to the energy $(\lambda=\partial J / \partial E)$, we find:

$$
D_{1}=\frac{1}{2 \pi} \frac{\partial J}{\partial q}=-\frac{\lambda^{3}}{4 \pi^{2}}\left(\frac{\partial \lambda}{\partial E}\right)^{-1}
$$

so that the sign of $D_{1}$ is opposite to that of $\partial \lambda / \partial E$ : there is coarsening $(D<0)$ if and only if the wavelength is an increasing function of the energy of the particle or, equivalently, of the amplitude $A$, which is related to the energy by $E=V(A)$.

Conserved equations - We now apply the same procedure to a class of conserved equations, which - in the same spirit - will be called generalized Cahn-Hilliard $(\mathrm{CH})$ equations:

$$
\partial_{t} u=-\partial_{x}^{2}\left[\partial_{x}^{2} u-F(u)\right] \equiv-\partial_{x}^{2} L[u] .
$$

The linear stability analysis of the trivial solution $u=0$ gives the spectrum $\omega=q^{2}-q^{4}$ and allows to define the fastest growing mode, $\lambda_{c}=\sqrt{2} \lambda_{0}$. We skip the calculations to attain the phase equation and just give the final expression for the phase diffusion coefficient,

$$
D=\frac{q^{2} \partial_{q}\left\langle q\left(\partial_{\phi} u_{0}\right)^{2}\right\rangle}{\left\langle u_{0}^{2}\right\rangle} \equiv \frac{q^{2} D_{1}}{\tilde{D}_{2}} .
$$

The $q^{2}$ factor at the numerator is distinctly due to the conservation law, i.e. to the operator $-\partial_{x}^{2}$ in front of $L$ in Eq. (10). The sign of $D$ is fixed by the quantity $D_{1}=$ $\partial_{q}\left\langle q\left(\partial_{\phi} u_{0}\right)^{2}\right\rangle$ as for the nonconserved case. Our criterion is therefore proved for the generalized $\mathrm{CH}$ equation as well.

Similarly to $J=\oint d \tau\left(\partial_{\tau} u_{0}\right)^{2}$ we can define $I=$ $\oint u_{0}^{2}(\tau)$, where $\oint d \tau$ means - in the mechanical analogythe time integral on an oscillation period. Using these quantities, $D_{2}=\left\langle\left(\partial_{\phi} u_{0}\right)^{2}\right\rangle=\lambda J / 4 \pi^{2}$ and $\tilde{D}_{2}=\left\langle u_{0}^{2}\right\rangle=$ $I / \lambda$. Finally, the diffusion coefficient $D$ reads

$$
\begin{array}{cl}
D=-\frac{\lambda^{2}}{J\left(\partial_{E} \lambda\right)} & \text { nonconserved (GL) models } \\
D=-\frac{\lambda^{2}}{I\left(\partial_{E} \lambda\right)} & \text { conserved (CH) models }
\end{array}
$$

The expressions clearly show that $D$ is negative if and only if $\partial_{E} \lambda>0$.

The different scenarios - Here above we have established for two important classes of evolution equations that the phase diffusion equation is stable (no coarsening) or unstable (coarsening), according to the sign of $d \lambda / d A$. Therefore, the following scenarios can be advanced (see Fig. 1). (i) If $\lambda(A)$ is a decreasing function the system typically develops a profile whose characteristic wavelength keeps constant in the course of time. This happens, e.g., if $F=-u-u^{3}$, i.e. $V(u)=u^{2} / 2+u^{4} / 4$. 

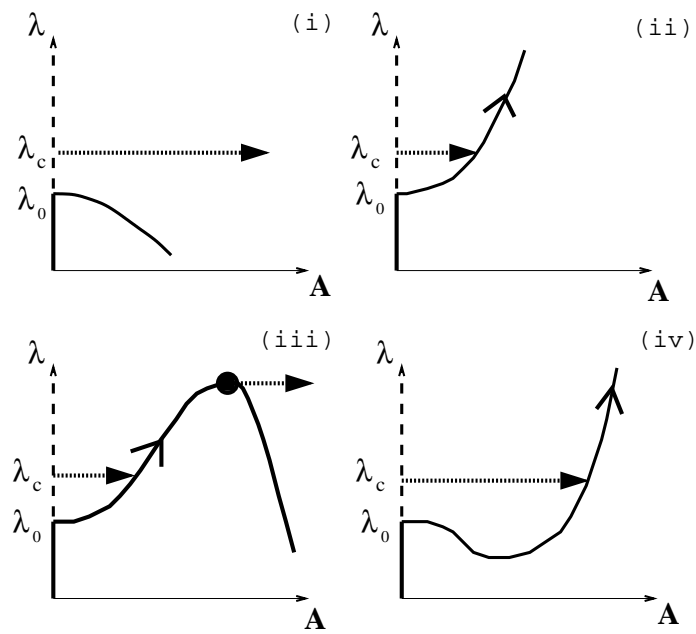

FIG. 1: Schematic wavelength-amplitude diagrams for the stationary solutions of period $\lambda$ and amplitude $A$. The four scenarios refer to different behaviors for $\lambda^{\prime}(A)$, as explained in the main text. The linear stability analysis of the trivial solution $u=0$ is represented by the line $A=0$ : stability (full line) for $\lambda<\lambda_{0}=2 \pi$ and instability (dashed line) for $\lambda>\lambda_{0}$. For the conserved models the flat interface starts to develop a profile of period $\lambda_{c}=\sqrt{2} \lambda_{0}$ (see the big dotted arrows close to the $\lambda$-axis). In cases (ii)-(iv) a coarsening process takes place, but in case (iii) it stops when $\lambda(A)$ attains the maximum (interrupted coarsening) and the amplitude starts growing (see the big dotted arrow close to the maximum).

(ii) If $\lambda(A)$ is an increasing function the system exhibits coarsening. This is what happens in the standard GL and CH models: $F=-u+u^{3}$. (iii) If $\lambda(A)$ starts to increase and afterwards it decreases the system is expected to display coarsening that stops at the maximum of $\lambda(A)$. This behavior is midway between (i) and (ii) and it is called interrupted coarsening. (iv) If $\lambda(A)$ starts to decrease and afterwards it increases the system essentially behaves as in (ii).

It is now interesting to consider a growth equation of physical interest, which does not fall into the classes we have discussed (see Ref. 8] for a general reference on crystal growth dynamics). The equation has the general form $\partial_{t} z=-\partial_{x} j$,

$$
\partial_{t} z(x, t)=-\partial_{x}\left\{B(m)+G(m) \partial_{x}\left[C(m) \partial_{x} m\right]\right\}
$$

where $m=\partial_{x} z$ is the local slope of the front and plays the role of $u$ in Eq. (10). It is worthnoting that Eq. (14) reduces to Eq. (10) if $C(m)$ and $G(m)$ are constant. Equations of this kind are often met in nature, such as in epitaxial growth of high symmetry as well as vicinal surfaces [8, 9]. They are also encountered in the study of sand ripples [10], in problems of spinodal decomposition, in the study of many linear defects, and so on.

It is possible to define the new variable $M(m)=$ $\int^{m} d s C(s)$, so that the stationarity condition $j=0$ can be rewritten as $B(M)+G(M) \partial_{x x} M=0$. Steady states therefore correspond to the solution of the Newton's equation for a fictitious particle, moving in the potential

$$
V(M)=\int d M \frac{B(M)}{G(M)} .
$$

Here we are interested in the growth dynamics of stepped, vicinal crystal surfaces [11] by vapor phase deposition techniques. With reference to Eq. (14), $m$ is the local slope of the step and [12]:

$$
\begin{aligned}
& B(m)=\frac{m}{1+m^{2}}, G(m)=\frac{1+\beta \sqrt{1+m^{2}}}{(1+\beta)\left(1+m^{2}\right)} \\
& C(m)=\frac{1+c\left(1+m^{2}\right)\left(1+2 m^{2}\right)}{(1+c)\left(1+m^{2}\right)^{3 / 2}}
\end{aligned}
$$

where $\beta$ and $c$ are positive, adimensional parameters. The former, $\beta$, is the relative strength between line diffusion and terrace diffusion, and the latter, $c$, is the strength of the elastic coupling between steps. The linear spectrum reads $\omega=q^{2}-q^{4}$. We now report the results of our analysis of $\lambda(A)$ for the different values of the parameters $\beta$ and $c$ (see also Fig. 1).

If elastic interactions are absent, $c=0, \lambda$ is always a decreasing function of $A$ (regime (i)). If elastic interactions are weak, $0<c<1 /(1+2 \beta), \lambda(A)$ decreases at small slopes and increases at large slopes (regime (iv)). For strong elastic interactions, $c>1 /(1+2 \beta), \lambda(A)$ is always an increasing function (regime (ii)).

From a dynamical point of view, we therefore expect no coarsening for $c=0$ and perpetual coarsening for $c \neq 0$. The case without elastic interactions has been considered in Ref. [13] for $\beta=0$ and in Ref. 9] for any $\beta$ : Refs. 9, 13] clearly show the absence of coarsening and the development of a constant pattern length scale with $\lambda=\lambda_{c}$. The case $c \neq 0$ has been treated in Ref. 14] (see Figs. 1,2 therein) and a persistent coarsening is revealed, both for $c<1 /(1+2 \beta)$ (regime (iv)) and for $c>1 /(1+$ $2 \beta$ ) (regime (ii)).

Recently, Eq. (14) has been modified in order to take into account anisotropy effects [15]. In this case, $\lambda(A)$ may present a maximum, which leads to regime (iii). As expected by our criterion, the coarsening is found [15] to interrupt at the maximum of $\lambda(A)$, where the amplitude grows unstably without bound.

An interrupted coarsening process was also found in a discrete model describing the growth dynamics of a onedimensional surface, the so-called Zeno model [16]. The surface is described as a sequence of steps which move following the Burton, Cabrera and Frank dynamics [17]. What is relevant in our context is that the interruption of coarsening at $\lambda=\lambda^{*}$ is related to the disappearance of stationary configurations for $\lambda>\lambda^{*}$ 16] (Fig. 1, scenario (iii)). 
Lyapunov functional - It is well known [18] that the two classes of nonconserved, Eq. (11), and conserved, Eq. (10), models can be derived by the Lyapunov functional 19] $\mathcal{F}[u(x, t)]=\int d x\left[\frac{1}{2}\left(\partial_{x} u\right)^{2}-V(u)\right]$, through the relations $\partial_{t} u=-(\delta \mathcal{F} / \delta u)$ (GL models) and $\partial_{t} u=$ $\partial_{x}^{2}(\delta \mathcal{F} / \delta u)$ (CH models). It is also easy to check that $d \mathcal{F} / d t \leq 0$ in both cases, i.e. dynamics proceeds so as to minimize $\mathcal{F}$.

It is trivial that $d \mathcal{F} / d t$ vanishes for the stationary configurations of period $\lambda, d_{t} \mathcal{F}\left[u_{\lambda}(x)\right]=0$. Rather, we are interested here to study the dependence of $\mathcal{F}$ on the pe$\operatorname{riod} \lambda$ of the steady state. The relation $\mathcal{F}\left[u_{\lambda}(x)\right] / \ell=$ $(J / \lambda-E)$ is found, where $\ell$ is the length of the growing front, and the derivative is easily evaluated [20], $\ell^{-1}\left(d \mathcal{F}\left[u_{\lambda}(x)\right] / d \lambda\right)=-\left(J / \lambda^{2}\right)<0$. This result, together with the relation $d \mathcal{F} / d t \leq 0$ which ensures that dynamics minimizes $\mathcal{F}$, complements the scenarios described in Fig. 1: if $\lambda$ increases with $A$ there is coarsening, and if $\lambda(A)$ has a maximum at $\lambda=\lambda^{*}$ coarsening stops at $\lambda^{*}$.

Perspectives - Our analysis has focused on equations where either coarsening takes place, or the wavelength is frozen while the amplitude 'diverges' depending on whether $\lambda(A)$ is an increasing or decreasing function. This does not exhaust all possible scenarios that occur in nonequilibrium systems. A prominent example is the Kuramoto-Sivashinsky (KS) equation which is known to produce spatio-temporal chaos with a dominant length scale (corresponding to the fastest growing mode). It would therefore be desirable to see how the steady-branch $\lambda(A)$ would behave in the presence of KS-dominated dynamics. We have done a preliminary study 21] of the modified $\mathrm{CH}$ equation [22]: $\partial_{t} u=-\partial_{x}^{2}\left[\partial_{x}^{2} u+u-u^{3}\right]+$ $\nu u \partial_{x} u$. For $\nu=0$ this equation reduces to the $\mathrm{CH}$ one, while for large $\nu$ an appropriate rescaling leads to the KS one. Above a critical value $\nu_{0}$ the branch $\lambda(A)$ undergoes a fold singularity where $\lambda(A)$ exhibits a turning point. We have found that in correspondence of that an anticoarsening process appears, that is to say $\lambda(t)$ decreases. Our work is in progress and it aims to clarify possible relations between the sign of $\lambda^{\prime}(A)$ and the type of dynamics exhibited by the system.

A more general puzzling question is whether, for extended systems enjoying basic symmetries (translation in the plane, $x \rightarrow x+x_{0}$ and $u \rightarrow u+u_{0}$ (or $z \rightarrow z+z_{0}$ ), and parity) coarsening, chaos or 'diverging' amplitudes with a frozen periodicity are the only possible scenarios. Is there any simple link between symmetries and the kind of dynamics (order or disorder) that a system exhibits when it does not succumb coarsening?

A final question concerns the diffusion coefficient $D$ when (perpetual) coarsening occurs, $D<0$. In this case, the typical pattern length scale $\lambda$ increases in time. Is it possible to derive the coarsening law, $\lambda(t)$, from the knowledge of $|D(\lambda)|$ ? It will be an important task to clarify all the above questions and to consider the possible extension of our arguments to higher dimension as well as to nonlocal equations, as they arise for example in solidification and viscous fingering.

We gratefully acknowledge useful discussions with several members of the Florentine Dynamics of Complex Systems group.

* Electronic address: politi@ifac.cnr.it

† Electronic address: chaouqi.misbah@ujf-grenoble.fr

[1] M. C. Cross and P. C. Hohenberg, Rev. Mod. Phys. 65, 851 (1993).

[2] Y. Kuramoto, Chemical oscillations, waves and turbulence (Springer, Berlin, 1984).

[3] In principle a negative $D$ could entail a decreasing of $\lambda$ (splitting) as well. However, this is inconsistent with the result $d \mathcal{F} / d \lambda<0$ (see the Section on the Lyapunov functional) and with the stability of the flat interface at small lengthscales.

[4] Noise does not modify the coarsening scenarios discussed throughout the paper.

[5] D. Zwillinger, Handbook of differential equations (Academic Press, San Diego, 1989), p.5.

[6] This is due to the Fredholm alternative theorem. See Ref. [5], p.13.

[7] H. Goldstein, C. Poole, and J. Safko, Classical Mechanics (Addison Wesley, San Francisco, 2002).

[8] P. Politi, G. Grenet, A. Marty, A. Ponchet, and J. Villain, Phys. Rep. 324, 271 (2000).

[9] F. Gillet, O. Pierre-Louis, and C. Misbah, Eur. Phys. J. B 18, 519 (2000).

[10] Z. Csahok, C. Misbah, F. Rioual, and A. Valance, Eur. Phys. J. E 3, 71 (2000).

[11] A vicinal surface is a surface which is slightly miscut from a high symmetry orientation. It looks like a flight of stairs with steps of atomic height separating large terraces.

[12] F. Gillet, Dynamique non linéaire de surfaces vicinales hors de l'équilibre. Ph. D. Thesis (Université de Grenoble, 2000).

[13] O. Pierre-Louis et al., Phys. Rev. Lett. 80, 4221 (1998).

[14] S. Paulin et al., Phys. Rev. Lett. 86, 5538 (2001).

[15] G. Danker, O. Pierre-Louis, K. Kassner and C. Misbah, Phys. Rev. E 68, 020601 (2003).

[16] P. Politi and J. Villain, Phys. Rev. B 54, 5114 (1996).

[17] W. K. Burton, N. Cabrera, and F. C. Frank, Phil. Trans. Roy. Soc. 243, 299 (1951).

[18] P. M. Chaikin and T. C. Lubensky, Principles of condensed matter physics (Cambridge University Press, Cambridge, 1995)

[19] $\mathcal{F}$ is usually written in terms of $U(u)=-V(u)$, but here is more useful to use $V(u)$.

[20] The terms $\lambda^{-1}(d J / d \lambda)$ and $d E / d \lambda$ cancel out.

[21] P. Politi and C. Misbah, unpublished.

[22] A. A. Golovin, A. A. Nepomnyashchy, S. H. Davis, and M. A. Zaks, Phys. Rev. Lett. 86, 1550 (2001). 